\title{
One-Year Follow-Up After Primary Coronary Intervention for Acute Myocardial Infarction in Diabetic Patients. A Substudy of the STENT PAMI Trial
}

\author{
Luiz Alberto Mattos, Cindy L. Grines, J. Eduardo de Sousa, Amanda G. M. R. Sousa, G regg W. Stone, \\ David Cox, Eulogio Garcia, Marie-Claude Morice, William $O$ 'N eill, Lorelei G rines, Judith Boura
}

São Paulo, SP, Brazil - Royal Oak, MI, Washington, DC, Charlotte, NC, EUA - Paris, França - Madrid, Espanha

Objective - This analysis was undertaken to determine the composite incidence of cumulative adverse events (death, reinfarction, disabling stroke, and target vessel revascularization) at the end of the first year after acute myocardial infarction, in diabetic patients who underwent coronary stenting or primary coronary balloon angioplasty.

Methods - From the STENT PAMI trial, we analyzed the 6-month angiographic and 1-year clinical outcomes of 135 diabetic (112, noninsulin dependent) patients who underwent the randomization process of the trial and compared them with 758 nondiabetic patients.

Results - Coronary stenting did not significantly reduce the primary composite clinical end point when compared with PTCA (20vs. 30\%, $p=0.2)$. A significant benefitfrom stenting was observed in patients with noninsulin dependent diabetes, with a trend toward a lesser need for new revascularization procedures $(10 \mathrm{vs.} 21 \%, p<.001)$, with a significant reduction in the primary composite clinical end point at 1 year (12vs. $28 \%, p=.04)$. At6 months, the restenosis rate were significantly reduced only in nondiabetic patients (18vs. $33 \%, p<.001)$. Diabetic patients had the same restenosis rate (38\%) either with stenting or balloon PTCA.

Conclusions - Coronary Stenting in diabetics noninsulin dependent offered a significant reduction in the composite incidence of major clinical adverse events compared with balloon PTCA.

Key words: myocardial infarction, stents, diabetes mellitus

Instituto Dante Pazzanese de Cardiologia, William Beaumont Hospital, Washington Hospital Center, Presbyterian Hospital, L'Institut Cardiovasculaire Paris Sud, Hospital Gregório Maranon

Mailing address: Luiz Alberto Mattos - Av. Jandira 550/121 - São Paulo, SP 04080-003 - Brasil - E-mail: pivmattos@uol.com.br
The STENT PAMI trial was a multinational randomized trial designed to compare the 6-month and 1-year clinical and angiographic outcomes of primary coronary balloon angioplasty (PTCA) and the routine implantation of coronary stents for acute myocardial infarction (AMI) with less than 12 hours of symptom onset ${ }^{1,2}$.

From previous consecutive series of elective coronary stenting, the clinical presence of diabetes mellitus (DM) remains as an independent predictor of the occurrence of major adverse events at late follow-up ${ }^{3-6}$.

In these series of non-AMI patients, diabetic patients who underwent coronary stent implantation showed a significantly higher rate of major coronary events, including death and new target vessel revascularization rates (TVR) at 6 months, and these were even higher in patients with insulin dependent diabetes ${ }^{3-6}$.

However, the possible benefits from routine stenting in diabetic patients who undergo percutaneous coronary interventions in the first hours of AMI onset are still not clear. Little data exist that report the 1-year clinical and 6-month angiographic outcomes between of primary coronary stenting or balloon PTCA in diabetic patients with AMI ${ }^{7-9}$.

The objectives of this study were to compare the 1year clinical and 6-month angiographic outcomes of diabetic and nondiabetic patients randomized to routine coronary stenting or PTCA in the first 12 hours of AMI onset, who were included in the STENT PAMI randomized trial.

\section{Methods}

Patients were considered for the trial if they were $\geq 18$ years of age, had symptoms of myocardial infarction less than 12 hours prior to signing an informed consent and had either ST-T segment elevation $\geq 1 \mathrm{~mm}$ (in 2 or more contiguous leads) or new left bundle-branch block. Clinical exclusion criteria included prior administration of thrombolytics for 
the index infarction, current use of warfarin, stroke within 1 month, renal failure, cardiogenic shock, expected survival $\leq 1$ year, women with childbearing potential unless a recent negative pregnancy test existed, or no contraindications to aspirin, heparin, or ticlopidine. The diabetic patients were included and their diabetic status was noted on each individual case report form, according to the clinical screening. A specific note identified insulin dependent patients but not whether they were receiving regarding treatment with diet or oral medication.

The complete protocol of the heparin-coated stent randomized trial has already been published ${ }^{1,2}$. In summary, patients received aspirin $(250 \mathrm{mg}$ IV or $325 \mathrm{mg}$ nonenteric coated chewable, prescribed indefinitely), ticlopidine $500 \mathrm{mg}$ orally (prescribed during the first month after AMI in patients treated with stents), heparin 5,000 to 10,000 IU intravenously (the ACT must be higher that 350 seconds) and in the absence of contraindications, beta-blockers were recommended in the emergency room. Patients were taken immediately to the cardiac catheterization laboratory where coronary angiography and left ventriculography were performed using low-osmolar ionic contrast media. Once the coronary flow was established, the operator determined whether the infarct lesion qualified for randomization as a native coronary artery, if the reference segment diameter was 3.0- to $4.5-\mathrm{mm}$, and if the lesion could be covered with 1 one or 2 stents ( $15 \mathrm{~mm}$ in length) by visual analysis. Patients were excluded from randomization if the operator thought the patient would be better treated medically or surgically or if the vessel seemed to have a great likelihood of requiring a stent. Coronary stent implantation exclusions also included ostial left anterior descending or circumflex lesions that could affect the left main stem, large side branches $(>3.0 \mathrm{~m} \mathrm{~m})$, an excessively tortuous vessel, or massive calcification.

The protocol stent was a $15-\mathrm{mm}$ heparin-coated Palmaz-Schatz stent mounted on the stent sleeve delivery system (Cordis). High-pressure stent implantation ( $\geq 16$ atm) was recommended in all patients.

Administration of thrombolytics or abciximab was discouraged unless a complete failure was determined by the operator. After the procedure, intravenous heparin was stopped unless a suboptimal result, $>10 \%$ diameter stenosis (visual), residual thrombus, dissection, or no-reflow was detected by the operator.

Primary balloon angioplasty was performed in the standard fashion, with matching 1:1 balloon/reference vessel size, to obtain the lowest stenosis diameter (SD). The crossover to stent was only permitted in the presence of a $\mathrm{SD} \geq 50 \%$ or severe flow threatening dissections, not solved with prolonged inflation, with low pressure or after the use of slightly bigger balloons.

Clinical events were monitored throughout the hospitalization, with 1-, 6-, and 12-month outpatient visits at each site. At 6 months, prior to the performance of the protocol follow-up coronary angiography, investigators documented angina class, stress testing (if performed), or evidence of ischemia on the ECG.

All acute and follow-up coronary angiograms were obtained using standard acquisition guidelines and submitted to independent angiographic core laboratories (Washington Hospital Center, Washington DC and Cardialysis, Rotterdam, The Netherlands) for analysis. Quantitative coronary angiography (QCA) analysis was performed using the CAAS II system (Pie Medical, The Netherlands). Myocardial perfusion was graded using the Thrombolysis in Myocardial Infarction (TIMI) classification ${ }^{10}$.

The primary end point of the study was the 1-year cumulative composite incidence of death, nonfatal reinfarction, disabling stroke, or new ischemic TVR, either percutaneous reinterventions or bypass surgery, according to the presence or absence of DM, randomized to coronary stenting or balloon PTCA.

All deaths were computed. Reinfarction was defined as clinical symptoms (or new ECG changes) with creatinine kinase $(\mathrm{CK})$ re-elevation and elevated MB fraction. A disabling stroke was defined as a stroke resulting in significant limitations in daily activities or the inability to live independently. Ischemic TVR was required if one or more of these components of ischemia were determined, class II-IV angina, an abnormal functional test, or occurrence after reinfarction.

The results were displayed in absolute numbers and their percentages. Continuous variables were presented with their respective standard deviation value. For the comparison of categorical variables the chi-square test was used (if the expected count of one cell was less than 5 observations, Fishers' exact test was used). For the comparison of continuous variables, Student's $t$ test was applied and, for both, $\mathrm{p}$ values equal to or less than 0.05 were considered significant and reported.

\section{Results}

Of the 900 patients randomized (452 to the heparin-coated Palmaz-Schatz stent and 448 to PTCA), 893 were included in the diabetic outcome analysis. Seven were excluded because no diabetic status was provided on the case report form. The presence of diabetes was detected in $135(15.1 \%)$ AMI patients, and they were compared with 758 nondiabetic patients. They were randomized to routine coronary stenting ( $n=72$, diabetic, and $n=377$, nondiabetic) or to balloon PTCA ( $n=63$, diabetic, and n=381, nondiabetic).

The diabetic patients compared with nondiabetic patients were older (64 vs. 59 years, $\mathrm{p}<0.001$ ), with AMI occurring more frequently in females ( $37 \mathrm{vs} .23 \%, \mathrm{p}<0.001)$, with a longer door-to-balloon time $(151 \pm 98$ vs. $127 \pm 85 \mathrm{~min}$, $\mathrm{p}<0.001)$, and with more than half of them having hyperten$\operatorname{sion}(52$ vs. $40 \%, \mathrm{p}=0.01)$ (table I).

Adjunctive pharmacology treatment was similar for diabetic and nondiabetic patients (table II).

The angiographic variables (table III) demonstrated that the diabetic patients had a more extensive coronary artery disease ( 55 vs. $43 \%, \mathrm{p}=0.01$ ) however no difference in mean baseline left ventricular ejection fraction $(47 \pm 12 \%$ vs. $48 \pm 12 \%, \mathrm{p}=0.12$ ), when compared with nondiabetic patients. The infarct related artery treated was similar in both groups. 
The acute procedural outcome is displayed in Table IV. The crossover rate of coronary stenting related to suboptimal PTCA results was similar between diabetic patients (14\%) or nondiabetic patients (15\%). According to core lab analysis, re-establishment of TIMI 3 flow after stenting or PTCA was similar (92 and 91\%) for both groups. Nondiabetic patients who underwent coronary stent implantation did not achieve less TIMI 3 flow than patients who underwent PTCA did (89 vs. $93 \%, \mathrm{p}=0.10$ ). Primary stenting resulted in a significantly smaller final stenosis diameter, in both diabetic and nondiabetic patients, compared with PTCA $(\mathrm{p}<0.001)$.

The acute procedural results judged by the QCA demonstrated significant benefits from coronary stenting compared to with PTCA, both in the diabetic or nondiabetic group. Stenting provided a larger final minimal luminal diameter (MLD) with a greater acute gain. The vessel size was similar in diabetic patients treated with stents or PTCA. Only the nondiabetic patients treated with PTCA had a smaller vessel size compared with that in the stent group (table IV).

The number of 6-month cinefilms available for core laboratory analysis were similar for all patients (table V), 74\% for diabetic patents treated with stent, $78 \%$ for diabetic patients treated with PTCA, and $75 \%$ for nondiabetic patients treated with either percutaneous device.

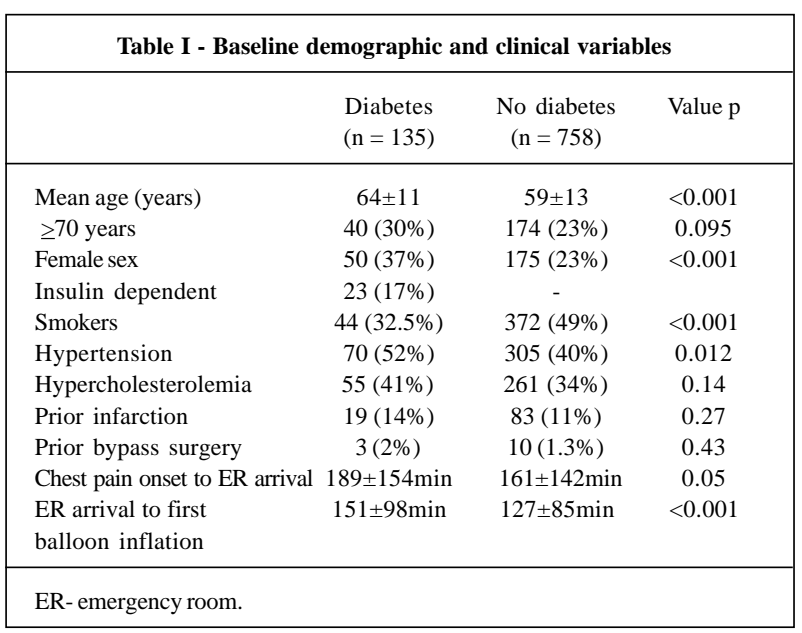

\begin{tabular}{|c|c|c|c|}
\hline & $\begin{array}{l}\text { Diabetes } \\
(n=135)\end{array}$ & $\begin{array}{c}\text { No diabetes } \\
(\mathrm{n}=758)\end{array}$ & Value $\mathrm{p}$ \\
\hline Aspirin & $132(98 \%)$ & $746(98 \%)$ & 0.49 \\
\hline Ticlopidine & $125(92.5 \%)$ & $678(89 \%)$ & 0.19 \\
\hline $\begin{array}{l}\text { Low-osmolar io } \\
\text { contrast media } \\
\text { Intravenous }\end{array}$ & $134(99 \%)$ & $736(97 \%)$ & 0.23 \\
\hline heparin & $135(100 \%)$ & $758(100 \%)$ & 1.00 \\
\hline Mean ACT & $437 \pm 127 \mathrm{~s}$ & $434 \pm 144 \mathrm{~s}$ & 0.82 \\
\hline Thrombolytics & 0 & $3(0.4 \%)$ & 1.00 \\
\hline Abciximab & $8(6 \%)$ & $38(5 \%)$ & 0.60 \\
\hline Beta-blockers & $79(58 \%)$ & $436(58 \%)$ & 0.83 \\
\hline
\end{tabular}

\begin{tabular}{|c|c|c|c|}
\hline \multicolumn{4}{|c|}{ Table III - Baseline angiographic data } \\
\hline & $\begin{array}{l}\text { Diabetes } \\
(\mathrm{n}=135)\end{array}$ & $\begin{array}{l}\text { No diabetes } \\
(\mathrm{n}=758)\end{array}$ & Value $p$ \\
\hline Vessel location & & & 0,94 \\
\hline Left anterior descending & $56(41,5 \%)$ & $317(42 \%)$ & \\
\hline Right coronary artery & $56(41,5 \%)$ & $338(44,5 \%)$ & \\
\hline Left circumflex & $21(15,5 \%)$ & $102(13 \%)$ & \\
\hline Bypass graft & $2(1,5 \%)$ & $1(0,5 \%)$ & \\
\hline $\begin{array}{l}\text { Multivessel } \\
\text { coronary disease }\end{array}$ & $74(55 \%)$ & $329(43 \%)$ & 0,014 \\
\hline $\begin{array}{l}\text { Mean left ejection } \\
\text { fraction }\end{array}$ & $47 \pm 12 \%$ & $48 \pm 12 \%$ & 0,12 \\
\hline
\end{tabular}

At the 6-month angiographic follow-up, diabetic stent patientshadasmallerreference diameter $(2.8 \pm 0.5 \mathrm{vs.} 3.0 \pm 0.5 \mathrm{~mm}$, $\mathrm{p}=0.04)$, similarminimal luminal diameter $(1.5 \pm 0.7 \mathrm{vs} .1 .4 \pm 0.7 \mathrm{~mm}$, $\mathrm{p}=0.4)$, and an absolutelateloss $(1.0 \pm 0.7 \mathrm{vs} .0 .7 \pm 0.8 \mathrm{~mm}, \mathrm{p}=0.11)$. The reduction in the SD DS in coronary stent patients was not statistical different toPTCA patients ( $44 \pm 23$ vs. $51 \pm 25 \%, \mathrm{p}=0.15$ ). Diabetic patients had the same restenosis rate for both percutaneous coronary strategies (38\%).

The nondiabetic stent patients had significant benefits in the 6-month QCA analysis. They had a similar reference diameter $(3.0 \pm 0.5$ vs. $3.0 \pm 0.6 \mathrm{~mm}, \mathrm{p}=1.00$ ), higher luminal diameter at follow-up $(1.9 \pm 0.7 \mathrm{vs} .1 .6 \pm 0.8 \mathrm{~mm}, \mathrm{p}<0.001)$, and also a lower $\mathrm{SD}(34 \pm 22$ vs. $44 \pm 23 \%, \mathrm{p}<0.001)$, compared with that in PTCA. Restenosis rates were significantly reduced (18 vs. $33 \%$, stent vs. PTCA, p<0.001).

In diabetic patients, the cumulative incidence of major complications after AMI was similar for both percutaneous strategies (table VI). Coronary stent implantation reduced by $29 \%$ the need for new ischemic TVR and by 33\% the occurrence of the combined incidence of major adverse events, although these reductions were not significant $(15 \mathrm{vs} .21 \%$, $\mathrm{p}=0.42$ and 20 vs. $30 \%, \mathrm{p}=0.21$, respectively).

When the clinical results at 1-year follow-up were analyzed according to the diabetic drug regimen (table VII), less ischemia-driven TVR procedures was noted in the coronary stenting strategy ( $10 \mathrm{vs.} 21 \%, \mathrm{p}=0.001)$, and also significant decrease in the cumulative incidence of combined events was then observed ( 12 vs. $28 \%, \mathrm{p}=0.04)$ in the noninsulin dependent patients $(n=112)$. In these patients, the restenosis rate were $37 \%$ for both percutaneous devices.

Nondiabetic patients treated with coronary stent implantation benefited with a significant reduction in the ischemia-driven TVR procedures ( $10 \mathrm{vs} .21 \%, \mathrm{p}<0.001)$ and in the combined clinical end point ( $17 \mathrm{vs.} 25 \%$, p $<0.005$ ). However, a routine stenting strategy promoted a higher death rate at 1-year follow-up in nondiabetic patients (6 vs. $3 \%$, $\mathrm{p}=0.05$ ), when compared with PTCA (table VI).

\section{Discussion}

The present data demonstrate the 1-year clinical and 6month angiographic outcomes of diabetic and nondiabetic patients who underwent primary percutaneous coronary interventions in the first 12 hours of AMI with coronary stenting or balloon PTCA with only rescue coronary stenting. 


\begin{tabular}{|c|c|c|c|c|c|c|}
\hline & \multicolumn{2}{|c|}{ Diabetes } & \multirow[t]{2}{*}{$\mathrm{p}$ Value } & \multicolumn{2}{|c|}{ No diabetes } & \multirow[t]{2}{*}{$\mathrm{p}$ Value } \\
\hline & $\begin{array}{c}\text { Stent } \\
(\mathrm{n}=72)\end{array}$ & $\begin{array}{l}\text { PTCA } \\
(\mathrm{n}=63)\end{array}$ & & $\begin{array}{c}\text { Stent } \\
(\mathrm{n}=377)\end{array}$ & $\begin{array}{c}\text { PTCA } \\
(\mathrm{n}=381)\end{array}$ & \\
\hline Stents deployed & $71(99 \%)$ & - & & $367(97 \%)$ & - & \\
\hline Crossover stents & - & $9(14 \%)$ & & - & $58(15 \%)$ & \\
\hline Number of stents & $1.3 \pm 0.6$ & $1.4 \pm 0.5$ & 0.41 & $1.4 \pm 0.6$ & $1.5 \pm 0.7$ & 0.20 \\
\hline TIMI flow post & & 1.00 & & 0.10 & & \\
\hline $0+1$ & $1(1.4 \%)$ & $1(1.6 \%)$ & & $2(0.6 \%)$ & $1(0.3 \%)$ & \\
\hline 2 & $5(7 \%)$ & $5(8 \%)$ & & $39(10.5 \%)$ & $25(7 \%)$ & \\
\hline 3 & $64(91.4 \%)$ & $57(90.5 \%)$ & & $332(89 \%)$ & $348(93 \%)$ & \\
\hline Balloon/artery ratio & $1.1 \pm 0.1$ & $1.2 \pm 0.2$ & 0.06 & $1.2 \pm 0.2$ & $1.2 \pm 0.1$ & 0.51 \\
\hline Final balloon pressure (ATM) & $15 \pm 3 \mathrm{~atm}$ & $9 \pm 4 \mathrm{~atm}$ & $<0.001$ & $15 \pm 4 \mathrm{~atm}$ & $9 \pm 3 \mathrm{~atm}$ & $<0.001$ \\
\hline DS post & $12 \pm 9 \%$ & $23 \pm 11 \%$ & $<0.001$ & $11 \pm 12 \%$ & $25 \pm 12 \%$ & $<0.001$ \\
\hline
\end{tabular}

\begin{tabular}{|c|c|c|c|c|c|c|}
\hline & \multicolumn{2}{|c|}{ Diabetes } & \multirow[b]{2}{*}{$\mathrm{p}$ Value } & \multicolumn{2}{|c|}{ No diabetes } & \multirow[b]{2}{*}{$\mathrm{p}$ Value } \\
\hline & Stent & PTCA & & Stent & PTCA & \\
\hline Acute results (mm) & $(\mathrm{n}=72)$ & $(\mathrm{n}=63)$ & $(\mathrm{n}=377)$ & $(\mathrm{n}=381)$ & & \\
\hline Reference diameter & $3.1 \pm 0.5$ & $2.9 \pm 0.6$ & 0.14 & $3.2 \pm 0.4$ & $3.0 \pm 0.4$ & $<0.001$ \\
\hline MLD Pre & $0.4 \pm 0.5$ & $0.4 \pm 0.6$ & 0.40 & $0.4 \pm 0.5$ & $0.4 \pm 0.5$ & 1.00 \\
\hline Post & $2.5 \pm 0.4$ & $2.1 \pm 0.4$ & $<0.001$ & $2.6 \pm 0.4$ & $2.1 \pm 0.5$ & $<0.001$ \\
\hline Acute gain & $2.1 \pm 0.7$ & $1.7 \pm 0.7$ & $<0.001$ & $2.2 \pm 0.7$ & $1.8 \pm 0.7$ & $<0.001$ \\
\hline DS post & $12 \pm 9 \%$ & $23 \pm 11 \%$ & $<0.001$ & $11 \pm 1 \%$ & $25 \pm 12 \%$ & $<0.001$ \\
\hline 6 months & $(\mathrm{n}=53)$ & $(n=49)$ & $(\mathrm{n}=285)$ & $(\mathrm{n}=285)$ & & \\
\hline Reference diameter & $2.8 \pm 0.5$ & $3.0 \pm 0.6$ & 0.04 & $3.0 \pm 0.5$ & $3.0 \pm 0.6$ & 1.00 \\
\hline MLD & $1.5 \pm 0.7$ & $1.4 \pm 0.8$ & 0.34 & $1.9 \pm 0.7$ & $1.6 \pm 0.7$ & $<0.001$ \\
\hline Late loss & $1.0 \pm 0.7$ & $0.7 \pm 0.8$ & 0.11 & $0.7 \pm 0.6$ & $0.5 \pm 0.7$ & $<0.001$ \\
\hline Net gain & $1.1 \pm 0.3$ & $1.0 \pm 0.4$ & 0.10 & $1.5 \pm 0.4$ & $1.3 \pm 0.4$ & $<0.001$ \\
\hline DS & $44 \pm 23 \%$ & $51 \pm 25 \%$ & 0.15 & $34 \pm 22 \%$ & $44 \pm 2 \%$ & $<0.001$ \\
\hline Restenosis & $20(38 \%)$ & $18(38 \%)$ & 0.95 & $50(18 \%)$ & $93(33 \%)$ & $<0.001$ \\
\hline Total occlusions & $3(6 \%)$ & $7(15 \%)$ & 0.11 & $13(5 \%)$ & $24(8 \%)$ & 0.06 \\
\hline
\end{tabular}

The noninsulin dependent diabetic patients who underwent coronary stenting in AMI demonstrated benefits from this strategy with a significant reduction in the combined incidence of occurrence of major adverse events at the 1-year clinical follow-up and a lower necessity for ischemiadriven TVR procedures. Only 23 insulin dependent patients were in the study, which does not allow for definitive conclusions to be made regarding this high-risk subgroup of diabetic patients.

Little data available regarding coronary stenting in diabetic patients during $\mathrm{AMI}^{7-9}$. In elective trials, the presence of DM had consistently been an independent predictor of a worse late outcome related to a higher restenosis rate and late loss index ${ }^{5,6,11}$, a faster atherosclerotic process, and a greater need for new revascularization procedures ${ }^{5,6,12}$.

Abizaid et al ${ }^{5}$ had have published results based on a larger sample size related to the late clinical follow-up of insulindependent diabetic patients who underwent coronary stenting. From a cohort of 248 patients with DM, 39\% of them were insulin-dependent and had the worst 6-month TVR rates $(28 \%, \mathrm{p}=<0.05)$, compared with nondiabetic patients $(16 \%)$ and diabetic patients who were noninsulin dependent $(18 \%)$.
The late clinical and angiographic results reported by Elezi et al ${ }^{6}$ in 3,554 consecutive patients who underwent coronary stenting, of whom $20 \%$ were diabetics, concluded that the presence of DM resulted in a less favorable clinical outcome at 1-year compared with the outcome in nondiabetic patients. The follow-up demonstrated a greater incidence of death, myocardial infarction, and repeat TVR procedures ( 23.5 vs. $18 \%, \mathrm{p}=0.001)$, and also higher restenosis rates, $36 \%$ in diabetic and $28 \%$ in nondiabetic patients $(\mathrm{p}=0.001)$.

The diabetic data results from the GUSTO II $b$ randomized trial (PTCA vs. accelerated TPA in AMI) have been published ${ }^{7.8}$. The diabetic group comprised $18 \%$ of the total 2,365 patients. This trial was not planned to test the coronary stent implantation strategy, but $9 \%$ of diabetic and $7 \%$ of nondiabetic patients received at least 1 stent as a bail-out to a suboptimal primary PTCA result. The 6-month incidence of composite major coronary events (death, reinfarction, and new TVR) were significantly reduced in patients who underwent coronary stenting, diabetic or not (19.3 and 21\%), compared to with patients who underwent PTCA (31.4 and $24.6 \%, \mathrm{p}=0.012$ ). The use of coronary stents most benefited the diabetic patients, providing them with a $39 \%$ reduction in 


\begin{tabular}{|c|c|c|c|c|c|c|}
\hline \multicolumn{7}{|c|}{ Table VI - Cumulative clinical events at 1-year follow-up } \\
\hline & \multicolumn{2}{|c|}{ Diabetes } & \multirow[b]{2}{*}{$\begin{array}{c}\mathrm{p} \text { Value } \\
\mathrm{P}\end{array}$} & \multicolumn{2}{|c|}{ No Diabetes } & \multirow[b]{2}{*}{$\mathrm{p}$ Value } \\
\hline & $\begin{array}{c}\text { Stent } \\
(\mathrm{n}=72)\end{array}$ & $\begin{array}{c}\text { PTCA } \\
(\mathrm{n}=63)\end{array}$ & & $\begin{array}{c}\text { Stent } \\
(\mathrm{n}=377)\end{array}$ & $\begin{array}{c}\text { PTCA } \\
(\mathrm{n}=381)\end{array}$ & \\
\hline Death & $3(4 \%)$ & $3(5 \%)$ & 1.00 & $22(6 \%)$ & $11(3 \%)$ & 0.05 \\
\hline Reinfarction & $1(1.4 \%)$ & $2(3 \%)$ & 0.60 & $12(3.2 \%)$ & $9(2.4 \%)$ & 0.49 \\
\hline Disabling stroke & 0 & $1(1.6 \%)$ & 0.47 & $2(0.5 \%)$ & $1(0.3 \%)$ & 0.62 \\
\hline Ischemic TVR & $11(15 \%)$ & $13(21 \%)$ & 0.42 & $36(10 \%)$ & $80(21 \%)$ & $<0.001$ \\
\hline Combined events & $14(20 \%)$ & $18(30 \%)$ & 0.21 & $62(17 \%)$ & $92(25 \%)$ & $<0.005$ \\
\hline
\end{tabular}

\begin{tabular}{|c|c|c|c|c|c|c|}
\hline \multicolumn{7}{|c|}{ Table VII - Clinical events at 1 year outcome in the diabetic patients according to the use of insulin } \\
\hline & \multicolumn{2}{|c|}{ Non-insulin dependent } & \multicolumn{4}{|c|}{ Insulin dependent } \\
\hline & $\begin{array}{c}\text { Stent } \\
(\mathrm{n}=60)\end{array}$ & $\begin{array}{c}\text { PTCA } \\
(\mathrm{n}=52)\end{array}$ & $\mathrm{p}$ Value & $\begin{array}{c}\text { Stent } \\
(\mathrm{n}=12)\end{array}$ & $\begin{array}{c}\text { PTCA } \\
(\mathrm{n}=11)\end{array}$ & $\mathrm{p}$ Value \\
\hline Death & $1(2 \%)$ & $2(4 \%)$ & 0.60 & $2(17 \%)$ & $1(9 \%)$ & 1.00 \\
\hline Reinfarction & 0 & $1(2 \%)$ & 0.46 & $1(8 \%)$ & $1(9 \%)$ & 1.00 \\
\hline Disabling stroke & 0 & $1(2 \%)$ & 0.46 & 0 & 0 & - \\
\hline Restenosis & $17 / 46$ & $14 / 38$ & 0.99 & $3 / 7$ & $4 / 9$ & 1.00 \\
\hline$(37 \%)$ & $(37 \%)$ & & $(43 \%)$ & $(44 \%)$ & & \\
\hline Ischemic TVR & $6(10 \%)$ & $11(21 \%)$ & 0.001 & $5(42 \%)$ & $2(18 \%)$ & 0.37 \\
\hline Combined events & $7(12 \%)$ & $14(28 \%)$ & 0.04 & $7(58 \%)$ & $4(36 \%)$ & 0.29 \\
\hline
\end{tabular}

the total sum of combined clinical events at 6-month followup. Nondiabetic patients who underwent stenting experienced a lesser gain, $16 \%$, compared with that in PTCA.

The comparison of these results with those from the Stent PAMI trial, which had a greater use of coronary stents, demonstrates similar benefits in the proportion of the reduction of major clinical events in diabetic patients, but an opposite finding in nondiabetic patients. In a longer clinical follow-up period (1 year), the reduction in the occurrence of major adverse clinical events were pretty much similar for diabetic or nondiabetic patients treated with stents (33 and $32 \%$, respectively) compared with those treated with PTCA. However, the differences in sample size, which was smaller in the diabetic patients, prevents the achievement of a statistical benefit, found only in nondiabetic stent patients.

The angiographic follow-up demonstrated that primary stenting in diabetic patients did not prevent them from having a higher restenosis rate (38\%), twice as high compared with that in nondiabetic patients $(18 \%, \mathrm{p}<0.001)$.

The comparison of QCA results obtained after coronary stenting demonstrated that nondiabetic patients keep their significant advantage obtained acutely until the 6month follow-up angiographic analysis, with a significantly lower SD. Diabetic stent patients had significantly similar acute procedural gains compared with PTCA patients but loose this gain during the healing process, keeping only a trend toward a significantly lower SD. The explanation is related to their higher late absolute loss than nondiabetic patients ${ }^{3,12}$. This evidence has already been observed in in- travascular ultrasound studies ${ }^{13,14}$. Diabetic patients developed greater intimal hyperplasia with significantly greater late lumen loss especially when stents were implanted, compared with nondiabetic patients ${ }^{3,4,12}$.

The major complications related to AMI (death, reinfarction, and stroke) were not reduced with coronary stent implantation compared with the PTCA strategy, either in the GUSTO II $b^{7}$ or in the Stent PAMI data.

In STENT PAMI, the nondiabetic patients who underwent a primary coronary stenting had a higher death rate $(\mathrm{p}=0.05)$, compared with those who underwent PTCA, at 1year follow-up ${ }^{15}$. In the diabetic patients, this difference did not occur. The trend toward an acutely decreased TIMI 3 coronary flow, observed in the nondiabetic stent arm, may have contributed to this finding.

Contrary to the Elezi ${ }^{6}$ results, obtained in elective coronary stenting, diabetic patients had significantly higher 30-day ( 1.4 vs. $2.7 \%, \mathrm{p}=0.01)$ and 1 -year death rates (3.8 vs. $8.3 \%, \mathrm{p}=0.001$ ), compared with those in nondiabetic patients. In our analysis of diabetic patients with AMI stenting, the 1-year cumulative death rate was similar. In the Stent PAMI baseline characteristics, the diabetic patients presented with many more adverse variables, clinical and angiographic, when compared with nondiabetic patients, exposing them potentially to a higher risk of death. Despite that, this did not occur. The higher rate of re-establishment of final TIMI grade flow 3 in the diabetic stent group may explain the equalization of the death rates, compared with those in nondiabetic patients. 
Abizaid et al ${ }^{5}$ analyzed the clinical results in diabetic patients treated with coronary stents and demonstrated a significantly higher death rate at hospital discharge but only for insulin-dependent patients ( 0 vs. $2 \%, \mathrm{p}<0.05$ ). However at 1 year follow-up, the death rates were similar for all diabetic patients, insulin-dependent or not (2.1 vs. $2.8 \%, \mathrm{p}=\mathrm{NS}$ ).

Diabetes mellitus has always been a subset analyzed in larger trials, either elective or AMI studies. Evidence obtained in this way may not have the same statistical power as that obtained from a specific planned study, regarding an undersized population. Despite that, all the conclusions related to the presence of DM and its long-term complications, such as the accelerated development of coronary heart disease, a higher rate of major clinical events and worse QCA findings after percutaneous coronary interventions, came from subgroup data analyses, and the majority of these reiterated the same poor late outcome of these patients ${ }^{4-8,11,16}$.

The protocol of the study did not recommend the use of intravenous abciximab during the percutaneous procedure, which is the reason for a low mean 5 percent usage. A substudy of the EPISTENT trial ${ }^{16}$ reported the diabetic nonAMI patient results (491 diabetics with 1-year clinical follow-up plus 127 patients with 6-month angiographic outcomes), demonstrating a more than 50 percent reduction in the need for new revascularization procedures for the target vessel at the end of the 6-month follow-up period. This was observed in the randomized group of coronary stenting plus routine abciximab infusion. In the 6-month angiographic follow-up, the authors found that diabetic patients treated with stents plus abciximab had a striking net gain in lumen diameter ( $62 \%$ of absolute gain vs. that in the nonabciximab arms), obtaining similar angiographic results as those in nondiabetic patients. The clinical consequences of this fact reflected positively in the TVR rates. However, the authors did not clarify the objective reason for these phenomena ${ }^{16-18}$. A new series may or may not confirm this previous finding.

Concluding, at 1-year clinical follow-up, coronary stent implantation in diabetic noninsulin dependent AMI patients offered a significant reduction in the cumulative incidence of major clinical adverse events, also less need for ischemia-driven TVR procedures. The 6-month angiographic results revealed that the restenosis rate was not modified with this strategy.

In nondiabetic patients, the 1-year clinical outcome after coronary stent implantation-revealed a significant reduction in the ischemia-driven TVR procedures with a $45 \%$ reduction in the restenosis rate, but with a higher death rate, compared with that in patients undergoing balloon PTCA.

\section{Acknowledgment}

A list of the names of the study's organizing committee members, comprising international investigators and the sponsors, has already been published in references 1 and 2 .

\section{References}

1. Grines CL, Cox D, Stone GW, et al. Coronary angioplasty with or without stent implantation for acute myocardial infarction. NEngl J Med 1999; 341: 1949-56.

2. Mattos L, Grines CL, Cox D, et al. Análise comparativa entre o implante primário de stents, angioplastia coronariana ótima e não ótima com cateter balão no infarto agudo do miocárdio. Resultados aos seis meses de uma subanálise do estudo STENT PAMI. Arq Bras Cardiol 2000; 75: 499-507.

3. Carrozza JP, Kuntz RE, Fishman RF, Baim DS. Restenosis after arterial injury caused by coronary stenting in patients with diabetes melito. Ann Intern Med 1993; 118: 344-9.

4. Van Belle E, Bauters C, Hubert E, et al. Restenosis rates in diabetic patients: a comparison of coronary stenting and balloon angioplasty in native coronary vessels. Circulation 1997; 96: 1454-60.

5. Abizaid A, Kornowski R, Mintz G, et al. The influence of diabetes melito on acute and late clinical outcomes following coronary stent implantation. J Am Coll Cardiol 1998; 32: 584-9.

6. Elezi S, Kastrati A, Pache J, et al. Diabetes Melito and the clinical and angiographic outcome after coronary stent placement. J Am Coll Cardiol 1998; 32: 1866-73.

7. Hasdai D, Granger C, Srivatsa S, et al. Diabetes Melito and Outcome after primary coronary for acute myocardial infarction: Lessons from the GUSTO-IIb angioplasty substudy. J Am Coll Cardiol 2000; 35: 1500-12.

8. Marso SP, Ellis SG, BhattDL, et al. The stenting in diabetics debate: insight from the large GUSTO IIb experience with extended follow-up. Circulation 1998; 98: I-78.

9. King SB. Acute myocardial infarction: Are diabetics different? J Am Coll Cardiol 2000; 35: 1513-5.
10. The TIMI Study Group: The Thrombolysis in Myocardial Infarction (TIMI) trial: Phase I findings. N Engl J Med 1985; 312: 932-6.

11. The Bypass Angioplasty Revascularization Investigation (BARI) Investigators: Seven-year outcome in the bypass angioplasty revascularization investigation (BARI) by treatment and diabetic status. J Am Coll Cardiol 2000; 35: 1112-9.

12. Aronson D, Bloomgarden Z, RayfieldEJ. Potential mechanisms promoting restenosis in diabetic patients. J Am Coll Cardiol 1996; 27: 528-35.

13. Kornowski R, Mintz G, Kent KM, et al. Increased restenosis in diabetes melito after coronary interventions is due to exaggerated intimal hyperplasia. A serial intravascular study. Circulation 1997; 335: 217-25.

14. Kornowski R, Mintz G,Lansky AH, et al. Paradoxic decreases in atherosclerotic plaque mass in insulin-treated diabetic patients. Am J Cardiol 1998; 81: 1298-304.

15. Grines CL, Cox D, Stone GW, et al. Stent PAMI: 12 month results and predictors of mortality. J Am Coll Cardiol 2000; 35: 402 A.

16. Lincoff AM,Califf RM, Moliterno DJ, et al. Complementary clinical benefits of coronary-artery stenting and blockade of platelet glycoprotein IIb/IIIa receptors. N Engl J Med 1999; 341: 319-27.

17. TopolEJ, MarkD, Lincoff AM, et al. Outcomes at 1 year and economic implications of platelet glycoprotein IIb/IIIa blockade in patients undergoing coronary stenting: results from a multicenter randomized trial. Lancet 1999; 354: 2019-24.

18. Neumann FJ, Kastrati A, Schmitt C, et al. Effect of glycoprotein IIb/IIIa receptor blockade with abciximab on clinical and angiographic restenosis after the placement of coronary stents following acute myocardial infarction. J Am Coll Cardiol $2000 ; 35: 915-21$ 